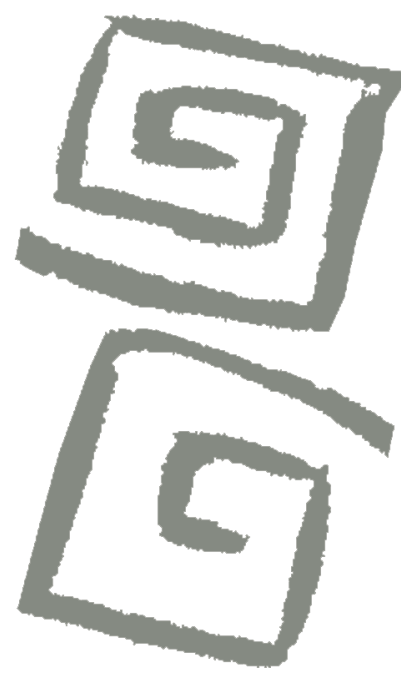

\title{
El estudio de la salud de los hombres desde una perspectiva de género: de dónde venimos, hacia dónde vamos
}

\author{
The study of men's health from a gender-based \\ perspective: where we come from, where we are going
}

Jorge Marcos-Marcos' ${ }^{1}$, José Tomás Mateos², Àngel Gasch-Gallén³, Carlos Álvarez-Dardet ${ }^{4}$

${ }^{1}$ Autor de correspondencia. Doctor en Estudios de Género. Docente Investigador, Grupo de investigación de Salud Pública, Universidad de Alicante, Alicante, España. $\triangle$ iD

${ }^{2}$ Doctor en Ciencias de la Salud. Investigador Postdoctoral, Departamento de Enfermería y Fisioterapia, Facultad de Enfermería y Fisioterapia, Universidad de Lleida, Lleida, España. $\triangle$ (iD

${ }^{3}$ Doctor en Ciencias de la Salud. Departamento de Fisiatría y Enfermería, Facultad de Ciencias de la Salud, Universidad de Zaragoza, Zaragoza, España. $\triangle$ iD

${ }^{4}$ Catedrático de Salud Pública. Docente

Investigador, Grupo de Investigación de Salud Pública, Instituto Universitario de Investigación de Estudios de Genero, Universidad de Alicante, Alicante, España. Investigador, Centro de Investigación Biomédica en Red de Epidemiología y Salud Pública (CIBERESP) Madrid, España. $₫$ iD
RESUMEN Llevar a cabo un análisis más integral y profundo de las diferencias y desigualdades en salud requiere de una aproximación más amplia al estudio de las masculinidades y la salud de los hombres en el momento actual. Estamos ante un tema cuyo interés ha ido a la par de la creciente preocupación por los riesgos y vulnerabilidades específicas de los hombres, pero también de la necesidad de involucrarlos en programas con capacidad de promover cambios positivos en el orden de género hacia la equidad en salud. Este artículo resitúa este campo dentro de la salud pública, proporcionando una visión amplificada sobre la salud de los hombres dentro del debate de los determinantes sociales de la salud y el análisis de las desigualdades. Sobre la base de un enfoque relacional de género, se formulan una serie de recomendaciones orientadas a las políticas y la investigación, que consideramos pueden contribuir a avanzar en el estudio y el desarrollo de programas desde una perspectiva de género en salud.

PALABRAS CLAVES Género y Salud; Salud de los Hombres; Masculinidades; Salud Pública.

\begin{abstract}
Comprehensive and in-depth analyses of differences and inequalities in health require a broad-based approach to the study of masculinities and men's health. Interest in this issue has grown in parallel to increased concern over specific risks and vulnerabilities faced by men, but also due to the need to involve them in programs capable of promoting progress towards gender-based health equity. This article attempts to reframe these issues from the perspective of public health, providing a wider viewpoint on men's health situated within debates on the social determinants of health and the analysis of health inequalities. Based on a relational gender approach, we formulate some recommendations regarding policy and research agendas, which we argue can contribute to advancing the study and development of programs from a gender-based perspective in health.
\end{abstract}

KEY WORDS Gender and Health; Men's Health; Masculinities; Public Health. 


\section{INTRODUCCIÓN}

La investigación sobre los hombres y las masculinidades es un campo de estudio relativamente reciente desde la perspectiva de género en salud. Sus bases conceptuales hunden sus raíces en una interesante confluencia de enfoques dentro de la teoría del género. Su eclosión puede situarse en la crítica al paradigma funcionalista inscrito en la teoría de la socialización de los roles sexuales; conceptualización que limitaba la comprensión del género al análisis de rasgos individuales de personalidad $^{(1)}$. Este debate contribuyó a mostrar que la masculinidad tenía que ver con las relaciones de poder entre hombres, no solo entre hombres y mujeres, abordando la masculinidad como una configuración de prácticas colectivas $^{(2)}$. La creciente influencia mundial de la cuarta ola del feminismo reafirma la idea de no entender "lo masculino" solo como una opción comportamental, sino como resultado de procesos de cambio cultural y aplicación de políticas de igualdad.

La teoría de las masculinidades, desde el punto de vista del constructivismo sociocultural, deriva también de los estudios críticos sobre etnicidad, que surgieron en EEUU, en la década de 1970, y que mostraron cómo la raza o la clase social son componentes esenciales de nuestras vidas sociales y políticas ${ }^{(3)}$. También en este sentido, estudios relacionados con el activismo feminista y de derechos de liberación afectivo-sexual originados en esa época aportan una base para el estudio crítico, desde una mirada posestructuralista de la sexualidad, las identidades, el sistema sexo-género ${ }^{(4)}$ y la práctica social del género $^{(5)}$. Algunos trabajos ya dan cuenta de los cambios en las relaciones de género entre mujeres y hombres, identificando nuevas posiciones de género en cuanto a temas como la intercambiabilidad de ciertas tareas, la responsabilización con algunas actividades más relacionadas con el poder por parte de las mujeres que anteriormente habían sido negadas, o la ritualización y exteriorización de aspectos como la seducción y el cuidado estético por parte de algunos hombres ${ }^{(6)}$. Estos cambios también sientan las bases para el desarrollo, por parte de diferentes disciplinas, de futuras preocupaciones sobre la salud de los hombres desde el análisis de las identidades, de cómo construyen sus masculinidades.

Una de las grandes conclusiones de la bibliografía científica reciente apunta a que el mayor riesgo de muerte prematura en los hombres es causado por factores evitables ${ }^{(7)}$. En aquellas sociedades que han experimentado profundos cambios sociodemográficos en un corto espacio de tiempo, el estudio de las identidades de género cobra un sentido especial. Nos permite un análisis más matizado de las diferencias y similitudes en los comportamientos y experiencias que influyen en la salud de las personas. El hecho de que a lo largo de la última década se haya ido configurando todo un corpus de evidencias orientado a entender la salud de los hombres desde su situación/posición y condición de género, no solo ha puesto de manifiesto la importancia de considerar las necesidades y particularidades biológicas de hombres y mujeres, sino que también ha supuesto un reconocimiento a la interacción de los factores socioculturales imbricados al género, tanto en la construcción de identidades como en la asignación de roles y responsabilidades, los cuales se reflejan en los estados de salud de ambos ${ }^{(8)}$. En el caso del estudio de las masculinidades, esto ha llevado a subrayar los riesgos y problemas de salud a los que se enfrentan como consecuencia de la interpretación del ejercicio de su identidad y mantenimiento de su posición social ${ }^{(9)}$.

Para la salud pública resulta fundamental conocer cómo opera el género influyendo en la salud. Con el auge del enfoque relacional, se empezó a poner de manifiesto que ciertas circunstancias sociales similares no solo pueden hacer vulnerables a hombres y mujeres, sino que también pueden producir diferentes efectos. Esto evidenció la necesidad de construir una base de conocimiento en torno a las diferencias tanto entre hombres y mujeres, como también entre los propios hombres y las propias mujeres ${ }^{(10,11)}$. Tomar en consideración un enfoque relacional en un sentido amplio implica concebir el género como un 
elemento que conecta, al mismo tiempo, las relaciones socioeconómicas, afectivas, simbólicas y de poder, y opera de manera simultánea a nivel intrapersonal, interpersonal e institucional ${ }^{(12)}$. Por tanto, obtener una visión más integral y profunda en el análisis de las diferencias y desigualdades en salud requiere una aproximación ampliada de la complejidad e implicaciones del estudio de las masculinidades y la salud de los hombres en el momento actual. Estamos ante un tema cuyo interés ha ido a la par, no solo a la creciente preocupación por las necesidades y riesgos específicos con relación a la salud de los hombres, sino también al reconocimiento de la necesidad de involucrarlos en programas con capacidad de promover cambios positivos en el orden de género hacia la equidad en salud.

\section{ACERCA DE ESTE ESTUDIO}

Este artículo tiene un objetivo doble: en primer lugar, (re)situar el estudio de los hombres y las masculinidades en los estudios de género y salud y, en segundo lugar, proporcionar una visión panorámica que contribuya a ampliar el campo visual desde el marco de los determinantes sociales y las desigualdades en salud. Para ello se toma como referencia un enfoque relacional, desde el convencimiento de que dar un paso más en el entendimiento de los procesos de salud-enfermedad-atención de las personas requiere atender a las sinergias que se establecen entre sus acciones.

Este artículo, basado en una revisión crítica de la bibliografía científica, nace de un proceso de confrontación reflexiva entre los autores para dar respuesta a una inquietud general: cómo el estudio de los hombres y las masculinidades puede contribuir a mejorar el conocimiento y abordaje de las diferencias y desigualdades de género en salud. Para ello, este artículo de naturaleza teórica se estructura en tres partes complementarias. En la primera, se realiza una aproximación al estudio de los hombres y las masculinidades desde una perspectiva de género; además, a modo de estado del arte, se proporcionan algunas claves sobre el papel de la teoría relacional de género a la hora de analizar los hombres y las masculinidades desde el marco de los determinantes sociales de la salud. En la segunda parte del artículo, se aborda, en primer lugar, las implicaciones del binomino riesgo e identidad de género, para después, en segundo término, proporcionar una visión ampliada en torno a los estudios críticos sobre los hombres, en base a explicaciones materialistas/estructurales, psicosociales y desde un enfoque de ciclo vital. Para facilitar el hecho de seguir el hilo conductor del artículo se insertan una serie de mensajes-resumen, que también contribuyen a vertebrar estas dos primeras partes. Por último, a lo largo de la tercera parte, adoptando un enfoque más pragmático, se formulan algunas recomendaciones para las políticas y la investigación que se consideran claves para seguir avanzando.

\section{GÉNERO Y SALUD EN EL ESTUDIO DE LOS HOMBRES Y LAS MASCULINIDADES}

Mensaje: Con el desarrollo de la teoría del género, y su progresiva incorporación a las políticas de salud pública, surge la necesidad de analizar la salud de los hombres sin perder de vista las interrelaciones con la salud de las mujeres.

Cada vez disponemos de un mayor corpus de conocimiento orientado a entender la salud de los hombres desde su posición dentro del orden de género ${ }^{(13)}$. Esto se hizo patente con la entrada del nuevo siglo, favorecido por el reconocimiento de la necesidad de prestar una mayor atención a su menor esperanza de vida, pero también al subrayarse el papel de las actitudes, expectativas y conductas de los hombres como factores causales de primer orden en las desigualdades en salud de las mujeres ${ }^{(14,15)}$. A continuación, se citan dos hechos puntuales que resultan ilustrativos de cómo el estímulo de las agendas políticas y de investigación ha contribuido a impulsar y reconceptualizar la salud de los hombres. 
En noviembre de 2001, un editorial del British Medical Journal, bajo el título “ ¿Están los hombres en peligro de extinción?", reflexionaba sobre un tema emergente en la bibliografía científica: el futuro de los hombres y su salud ${ }^{(16)}$. Uno de los aspectos centrales que ponían de manifiesto los autores es que, a pesar de tener la mayoría de los determinantes sociales de la salud en su favor, los hombres tienen mayores tasas de mortalidad. Ese mismo mes se celebraría en Viena el I Congreso Mundial sobre la Salud de los Hombres, que daría pie a la puesta en marcha de la Sociedad Internacional para la Salud de los Hombres. El citado editorial se hacía eco de estas iniciativas, expresando la esperanza de que sirvieran para relanzar la investigación sobre el papel y las implicaciones del género en la salud de las poblaciones. El segundo hecho remite al Ilamamiento de la Comisión de Determinantes Sociales de la Salud de la Organización Mundial de la Salud (OMS) a "construir un movimiento global por la equidad en salud"(17). Como parte de dicho movimiento, dentro del $54^{\circ}$ Periodo de Sesiones de la Comisión sobre la Condición Jurídica y Social de la Mujer, se abordaría de forma específica la necesidad de involucrar a los hombres en la consecución de la igualdad de género ${ }^{(18)}$.

Mensaje: La teoría relacional de género ha jugado un papel central en la consideración de los hombres dentro del marco de los determinantes sociales de la salud.

El desarrollo de paradigmas complementarios a los de la biomedicina ha permitido analizar los procesos de salud-enfermedadatención desde una visión más sensible a lo local y particular, interpretando la etiología desde la consideración a redes multicausales ${ }^{(19,20)}$. Si bien las diferencias en salud entre hombres y mujeres han sido explicadas durante mucho tiempo desde concepciones biológicas, asumiéndolas como naturales ${ }^{(21)}$, en la actualidad no se suele/debe caracterizar la enfermedad sin tomar en consideración las experiencias de las personas dentro del marco social en el que se desarrollan sus vidas $^{(22)}$. Dicha consideración a las singularidades subjetivas constituye una de las claves para el desarrollo del enfoque relacional de género en el corpus científico de la salud pública.

Estudiar el orden de género desde la teoría relacional, considerando la estrecha vinculación existente entre lo biológico y lo sociocultural ${ }^{(23)}$, lo convierte en un elemento fundamental en el estudio de las causas y los factores a través de los cuales las desigualdades de género se perpetúan en un determinado contexto. Esto ha contribuido a que el género sea principalmente descrito como una característica social, y no únicamente como una característica de los individuos. A pesar de las diversas formas de conceptualizar el género, podríamos establecer que estamos ante una estructura social con dos elementos fundamentales de análisis: diferencia/dicotomía versus desigualdad/asimetría ${ }^{(24)}$. Aunque ambas dimensiones son claves, la realidad es que el abordaje del binomio género-salud no cuenta con un único marco de referencia.

La idea del género como elemento relacionado con la salud emergió con fuerza en la bibliografía científica durante la segunda ola del feminismo, desarrollándose en primera instancia como preocupación sobre las necesidades de atención y la morbilidad diferencial de las mujeres. Esto llevó, principalmente, a criticar tanto el modelo androcéntrico de la medicina, como también la atención a la salud reproductiva y la medicalización de su abordaje $^{(25)}$. Por otro lado, desde mediados de la década de1970, se había empezado a poner de manifiesto que la conformidad con normas tradicionales sobre la masculinidad parecía predisponer a un incremento de los riesgos para la salud física y el empobrecimiento emocional ${ }^{(26,27)}$. Sobre la base de la teoría del rol sexual, esta corriente se centraría en afirmar que los hombres, desde edades tempranas, aprenden comportamientos que aumentan su exposición a accidentes mortales y a sufrir determinadas enfermedades ${ }^{(9)}$. A principios de la década de 1980, se empezaron a explorar -de forma más analítica- los factores sociales subyacentes a los procesos de salud-enfermedad de las mujeres. Esto llevó a poner el acento en 
las diferencias y desigualdades en el estado de salud, pero también en los sesgos de género en la investigación y la atención sanitaria ${ }^{(28)}$. Los estudios se diversificaron, centrándose en la forma en que hombres y mujeres percibían y experimentaban los procesos de salud-enfermedad, al tiempo que también empezaron a ser temas de interés la violencia contra las mujeres o los derechos de gais, lesbianas y personas transgénero. Esto supuso un impulso al desarrollo de investigaciones centradas en la salud de los hombres.

Durante la década de 1980, la creciente crítica a la teoría del rol sexual -fundamentalmente por enfatizar la importancia de la primera infancia en la socialización de género, omitiendo cuestiones relativas con las estructuras de privilegio- fue dando lugar a todo un corpus de conocimiento en torno a cómo los individuos interiorizan el género(1). De forma progresiva, el género empezó a ser incorporado como una categoría analítica sobre la diferencia sexual y las relaciones entre hombres y mujeres y, con ello, a ser tratado como determinante de la salud. Del mismo modo, esta década marcó un hito en las políticas e investigaciones en materia de género y salud ${ }^{(29)}$. A ello contribuyó la irrupción en la bibliografía científica de publicaciones como Gender and health: an update on hypotheses and evidence, un artículo seminal en el que Lois M. Verbrugge ${ }^{(30)}$ describía los principales patrones, hipótesis y explicaciones de las diferencias de género en salud en EEUU. Los resultados ponían de manifiesto que los hombres sufrían más enfermedades que ponían en riesgo sus vidas, las cuales conducen en mayor medida a situaciones de discapacidad permanente o muerte prematura. Resultados que serían corroborados por una serie de informes del Morbidity and Mortality Weekly Report, que aportaban evidencias de las diferencias en las tasas de mortalidad de hombres y mujeres de entre 15 y 24 años con relación a las seis principales causas de muerte en EEUU: accidentes de tráfico, suicidios, otros daños no intencionados, homicidios, cáncer y enfermedades del corazón ${ }^{(31)}$. Estas publicaciones contribuyeron a poner de manifiesto la necesidad de abordar el impacto e implicancias del género en la salud de los hombres.

Con todos estos aportes, el enfoque relacional precisa la existencia de prácticas reflexivo-corporales en cuanto al género, que surgen en las relaciones interpersonales, no como actos solamente internos e individuales, sino que construyen el mundo social. Así el género se debe analizar desde su multidimensionalidad y considerando que las relaciones se construyen sobre la base de procesos históricos y dinámicos ${ }^{(32)}$. Este enfoque aporta la idea de la incorporación de las exigencias de género para todas las personas, que son socializadas en el orden que dicha estructura establece y dentro de una jerarquía que provoca desigualdades en salud.

Mensaje: El hecho de que los factores biológicos no expliquen por sí solos por qué las brechas de género en salud cambian en el tiempo, y entre distintos grupos sociales, obliga a poner el foco en los factores de orden social subyacentes a los procesos de salud-enfermedad-atención.

A menudo los marcos explicativos suelen centrarse en la distribución de la salud y sus determinantes, el origen y las causas de las desigualdades en salud o los mecanismos que las mantienen. Sea como fuere, partimos de un supuesto clave: la salud de hombres y mujeres no solo es diferente, sino también desigual. Diferente porque existen factores biológicos que se manifiestan de manera distinta en la salud y en los riesgos de enfermar; desigual porque hay factores de orden social, en parte explicados por el género, que influyen de una manera injusta, por ser evitables, en la salud de las personas $^{(33)}$. Esto lleva a que el género no solo se entienda como un principio articulador de creencias, valores y costumbres, sino también de diferencias en la exposición y vulnerabilidad a factores de riesgo ${ }^{(21)}$. Vemos así cómo las diferencias biofisiológicas que existen entre mujeres y hombres modifican y moldean las respuestas de los cuerpos de las personas a los impactos del entorno sobre la propia salud.

En salud pública la investigación sobre el género toma como elemento central la 
relación que se establece entre género y poder $^{(34)}$. Sin embargo, tan erróneo es buscar la igualdad en la salud de hombres y mujeres, como asumir diferencias donde no existen ${ }^{(35)}$. Por otro lado, a la hora de analizar las diferencias y semejanzas en la salud de las mujeres y los hombres, resulta de interés tomar en consideración que rara vez son puramente de naturaleza biológica o de orden social ${ }^{(8)}$.

Desde un punto de vista biológico los hombres son considerados más vulnerables que las mujeres. Esta afirmación suele apoyarse en argumentos relativos a los niveles de estrógenos y a la diferente composición cromosómica, los cuales contribuyen a reducir el riesgo de las mujeres en las enfermedades del corazón mediante la reducción de la circulación de colesterol nocivo. Por su parte, la testosterona pone a los hombres en mayor riesgo de afecciones potencialmente mortales a causa de la inmunosupresión ${ }^{(36)}$. Sin embargo, los factores biológicos no explican por sí solos por qué las brechas de género en salud cambian en el tiempo y en distintos grupos sociales y contextos. Es por ello que, a la hora de analizar las desigualdades de género en salud, los estudios tienden a centrarse en los factores sociales que conforman el bienestar, la posición social y los patrones conductuales de hombres y mujeres.

La interrelación entre los factores biológicos y las relaciones de género se pone de manifiesto de múltiples formas, por ejemplo, al considerar la infección por $\mathrm{VIH} /$ sida de las mujeres a una edad más temprana que la de los hombres, o su mayor mortalidad por violencia de género ${ }^{(21)}$. Sin embargo, aunque las mujeres sufren más enfermedades y su salud es peor, tienen una esperanza de vida mayor a la de los hombres. Se trata de un fenómeno ampliamente documentado del que diversas revisiones proporcionan descripciones de su magnitud y alcance ${ }^{(37,38)}$. Las inequidades de género en morbilidad no son tan unívocas como las relativas a la mortalidad. La brecha entre hombres y mujeres varía según enfermedades específicas, atendiendo a la etapa del ciclo vital ${ }^{(39)}$. En las edades más jóvenes, los hombres tienden a involucrarse en comportamientos más perjudiciales para la salud, lo que aumenta el riesgo de muerte prematura por lesiones accidentales u homicidio ${ }^{(40)}$. Por el contrario, las mujeres son más propensas a sufrir procesos de naturaleza crónica, que no necesariamente potencian la muerte prematura, pero tienen un gran impacto negativo en su calidad de vida ${ }^{(41,42)}$.

A lo largo de los últimos años, con el desarrollo de distintos modelos conceptuales para explicar las desigualdades de género en salud, como es el caso del propuesto por la Red de Conocimiento sobre Mujeres y Equidad de Género, que se presenta vertebrado por procesos de retroalimentación entre causas, factores y consecuencias ${ }^{(43)}$, ha contribuido a poner de manifiesto que, abordar la cuestión de por qué los hombres tienen menor esperanza de vida pero padecen menos enfermedades crónicas, requiere tomar en consideración una combinación de condiciones y características biológicas, socioestructurales, psicosociales y comportamentales que diferencian sus vidas ${ }^{(44)}$. Analizar en términos de exposición o vulnerabilidad diferencial estas categorías explicativas, cobra sentido también en el caso de los hombres.

\section{LA CARA OCULTA DE LOS PRIVILEGIOS}

\section{Salud, riesgo e identidad de género}

Mensaje: Riesgo e identidad de género conforman un binomio clave en el análisis de las masculinidades y la salud de los hombres, pero requiere ir más allá de un enfoque de decisiones individuales, analizando la asunción de riesgos como resultado de procesos de interacción social.

Una de las principales aproximaciones al riesgo tiene que ver con las consecuencias de los "estilos de vida" (45). Sobre la base de los riesgos, la interpretación de los patrones de salud-enfermedad a menudo queda asociada a decisiones personales $y$, por tanto, a cuestiones de autocontrol y responsabilidad individual ${ }^{(46)}$. Abordado desde esta perspectiva, 
el análisis de los factores de riesgo tiende a minimizar el impacto de las estructuras sociales, económicas y políticas. Esto ha supuesto considerar que si se quiere comprender por qué una persona lleva a cabo determinados comportamientos de riesgo, debe tenerse en cuenta que más que una decisión racionalindividual es producto de un proceso de interacción social. Así, la asunción del riesgo puede ser considerada como una negociación con los discursos dominantes, en los que impera el modelo de masculinidad hegemónica, obligando a las personas a posicionarse, en el caso de muchos hombres, según sus exigencias. En esta negociación, el beneficio social esperado por la adscripción a la hegemonía disminuye las posibilidades del cambio hacia formas de comportamientos y relaciones más saludables.

Las consideraciones sobre el riesgo adquieren especial significación cuando van referidas a poblaciones jóvenes. Se trata de una etapa caracterizada por la búsqueda de referentes; aspecto fundamental a la hora de analizar el papel del riesgo en la configuración de la identidad de género de los hombres ${ }^{(47)}$. Esto adquiere mayor sentido cuando se toma en cuenta que los mayores riesgos para su salud son el resultado de factores y comportamientos que podrían ser modificados ${ }^{(15)}$. Es por ello que, solo desde una perspectiva de género se puede entender que la mayor mortalidad o siniestralidad de los hombres, o que utilicen menos los servicios sanitarios preventivos, tiene que ver con concepciones y comportamientos asociados a la forma en que se construyen como hombres, y se representan como dominantes ${ }^{(48,49)}$. Esto supone que el género se manifiesta principalmente en las transacciones inscritas en las actividades cotidianas ${ }^{(50)}$. Es aquí cuando la asunción de riesgos parece constituirse en una estrategia fundamental a la hora de cumplir con las expectativas de lo que se considera ser un hombre ${ }^{(8)}$.

La masculinidad ha sido fundamentalmente descrita en la bibliografía científica en sentido problemático ${ }^{(51)}$. Esto conduce de forma inevitable a la cuestión de por qué los hombres realizan en mayor medida conductas que ponen en riesgo su salud. Una perspectiva recurrente ha sido la proporcionada por Will Courtenay ${ }^{(52)}$. Su tesis apunta a que no solo algunas conductas de riesgo son culturalmente definidas como "masculinas", sino que pueden utilizarse como forma de definir la virilidad. Desde esta perspectiva, es la asociación con ciertos ideales de masculinidad lo que contribuye a algunas de las más importantes diferencias en salud entre mujeres y hombres ${ }^{(53)}$. Esto también ha llevado a describir ciertas formas de entender la masculinidad como un estado de precariedad social. Esta perspectiva teórica se fundamenta en dos principios básicos. Por un lado, la masculinidad es descrita como un estatus social que no solo debe ganarse, sino que puede perderse (en contraste con la condición de mujer, percibida como un estado que fluye al son de cambios biológicos y que, una vez obtenida, no se corre el riesgo de perder). Por el otro, la masculinidad es principalmente confirmada por los demás y, por tanto, requiere de demostraciones públicas ${ }^{(54)}$.

En el caso de las personas jóvenes, el proceso de construcción identitaria adquiere una mayor significación en torno a la idea del riesgo por tratarse de una etapa de la vida en la que no solo lo prohibido puede resultar atractivo, sino que el hecho de que los mensajes normativos suelen provenir de una autoridad a desafiar, puede invitar a transgredirlos ${ }^{(55)}$. Paralelamente a la concepción del riesgo en sentido negativo, a lo largo de los últimos años, se ha venido poniendo en valor una connotación positiva. Descrito como algo consustancial al proceso vital ${ }^{(56)}$, un componente esencial en el autoconocimiento y el desarrollo socioafectivo $y$, por consiguiente, en la definición de la propia identidad ${ }^{(57)}$. Es precisamente esta concepción de las identidades la que ha subrayado la importancia del género en el entendimiento de la sexualidad. La investigación ha destacado la vivencia de la masculinidad como un hecho social íntimamente ligado al ejercicio de la sexualidad, que no solo ha llevado a subrayar el papel de la heteronormatividad en el proceso de construcción identitaria, sino también la imbricación de los comportamientos de riesgo con el establecimiento de relaciones de poder ${ }^{(58)}$. De esta 
manera, las diferentes expectativas que el orden de género proyecta en relación a las conductas consideradas apropiadas, tienen una gran influencia en el comportamiento sexual de las personas ${ }^{(59)}$. Sin embargo, el dominio de ciertas nociones biomédicas, junto a los ideales neoliberales de las elecciones personales y los modelos psicológicos sobre el comportamiento individual han contribuido a subrayar discursos que pasan por alto la importancia del contexto y los determinantes sociales en la conformación de las relaciones y las experiencias en salud. Esto ha provocado que desde la corriente principal se fundamenten las diferencias en salud de los hombres sobre la base de comportamientos individuales. Aunque se trata de un enfoque de gran utilidad, en los últimos años se vienen desarrollando, de forma paralela, líneas de investigación en la que dichas explicaciones comportamentales son enmarcadas en un espectro mayor de variables determinantes de la salud.

\section{La salud desde los estudios críticos sobre los hombres y los marcos explicativos de las desigualdades}

\section{Mensaje: Dentro del análisis de las desigual- dades en salud, los estudios críticos sobre los hombres proporcionan una visión más amplia a la hora de caracterizar las masculinidades y estudiar la salud de los hombres atendiendo a explicaciones materialistas/estructurales, psi- cosociales y desde el enfoque del ciclo vital.}

El elemento común que comparten la perspectiva de género y la salud pública es su consideración social. Es precisamente la asunción de dicha dimensión social la que pone a la salud en un lugar destacado en cualquier discusión sobre equidad y justicia social ${ }^{(60)}$. Esto no solo obliga a tomar en consideración las desigualdades en mortalidad o en natalidad, sino también las desigualdades en oportunidades básicas, las desigualdades profesionales o las que giran en torno al hogar. Entendida de esta manera, la equidad de género tiene que ver con justicia en la distribución de beneficios y responsabilidades, elementos clave a la hora de entender las masculinidades.

En este sentido, los estudios críticos sobre los hombres, corriente que surgió del feminismo, y en la que pueden incluirse los movimientos político-sociales y académicos sobre la lucha por la diversidad afectivo-se$x$ al(61) $^{(61)}$ vienen proporcionando una visión amplificada al caracterizar las masculinidades y estudiar las interrelaciones que se establecen en la salud de hombres y mujeres. Se presenta a continuación una aproximación a dichos estudios, pero centrando el análisis en algunos de los enfoques explicativos de las desigualdades en salud recurrentes en la bibliografía científica.

\section{Explicaciones estructurales}

Desde este enfoque, sobre la base de la epidemiología social y política, en el análisis de las desigualdades en salud se pone énfasis tanto en los procesos económicos y decisiones políticas que condicionan el acceso a los recursos como en los elementos que conforman el complejo material de nuestras sociedades, especialmente, en las infraestructuras públicas (educación, servicios sanitarios, regulaciones de salud laboral, etc. $)^{(62,63)}$. De esta manera, la relación entre desigualdades en salud y desigualdades socioeconómicas estaría especialmente mediada por aspectos como el nivel de ingresos, el nivel formativo, el estatus profesional o la situación ocupacional ${ }^{(64)}$. Por tanto, las desigualdades de género en salud serían principalmente el resultado de las diferencias en la posición socioeconómica de hombres y mujeres. Una de las hipótesis explicativas apunta a que, en la medida en que exista convergencia en dicha posición socioeconómica, también debe haberla con relación al estado de salud ${ }^{(65)}$. Sin embargo, no estamos solo ante cuestiones de naturaleza puramente económica, sino también de poder y de capacidad de toma de decisiones. En este sentido, los roles y la división sexual del trabajo han jugado, y siguen jugando, un papel determinante. De ahí que estemos ante un enfoque que cobra especial sentido cuando el género es entendido como una estructura social. 
Desde una perspectiva crítica, los hombres son reconocidos como el grupo más favorecido económica y socialmente. No solo ocupan más cargos de poder en la mayoría de las categorías profesionales, especialmente en las ocupaciones de mayor prestigio social, sino que ostentan las condiciones laborales con mejores garantías para su salud ${ }^{(29,66)}$, incluyendo un menor riesgo de sufrir situaciones de pobreza a lo largo de sus vidas. Esto se debe, entre otras razones, a que es más probable que tengan empleos estables, que trabajen a tiempo completo y que reciban un salario mayor que las mujeres por un trabajo similar ${ }^{(67)}$. Del mismo modo, la incorporación de las mujeres al empleo remunerado no se ha traducido en una redistribución equitativa del trabajo reproductivo y de cuidados ${ }^{(68,69)}$.

Aunque se ha señalado que los hombres suelen tener menores redes de apoyo ${ }^{(70)}$, con el potencial de mejorar el bienestar y/o amortiguar el impacto negativo en situaciones adversas $^{(71)}$, la evidencia científica no es concluyente al respecto. De hecho, las mujeres, en mayor medida que los hombres, suelen reportar estresores crónicos ${ }^{(72,73)}$. En los hombres, no compatibilizar en la misma medida que las mujeres la esfera productiva y reproductiva, particularmente en lo que respecta a asumir responsabilidades de cuidado informal, disminuye sus riesgos de padecer ciertos problemas de salud, aumentando otros ${ }^{(74,75)}$. En esta misma línea, también son aún limitados los estudios que han comparado la salud de hombres pertenecientes a distintos grupos socioeconómicos.

Las explicaciones estructurales sobre las desigualdades han sido poco utilizadas a la hora de analizar la salud de los propios hombres. Quizá porque, mientras la desigualdad socioeconómica ha servido para explicar las desventajas en salud de las mujeres, no hay una teoría concluyente para explicar, por ejemplo, la menor esperanza de vida de los hombres ${ }^{(38)}$. Otra forma de ampliar el debate entre las desigualdades en salud y los estudios de los hombres es describir las explicaciones estructurales como una forma de representación simbólica de poder, de la masculinidad sobre la feminidad, sobre la base de variables relativas al nivel de ingresos y la acumulación de riqueza. Desde una perspectiva crítica, se pone de manifiesto que las asimetrías de poder existentes en las relaciones de género forman parte del "catálogo" de motivaciones subyacentes a ciertas actitudes y comportamientos que, a menudo, conducen a los hombres a dañar su propia salud ${ }^{(12)}$. Resulta de interés considerar la interrelación entre prácticas "generizadas" hegemónicas y prácticas institucionalizadas, como el hecho de tener que realizar largas jornadas laborales, hacer horas extras o no acogerse al derecho de permiso por paternidad; prácticas con implicaciones para la salud que refuerzan la división sexual entre trabajo productivo y reproductivo para hombres y mujeres respectivamente.

\section{Explicaciones psicosociales}

Esta corriente se centra en el análisis de las percepciones y experiencias personales en torno a los estados de estrés derivados de situaciones de desigualdad social. El estrés producido por el ambiente social en el que se vive altera las funciones neuroendocrinas, incrementando la vulnerabilidad del organismo a enfermar ${ }^{(76)}$. Las "exposiciones adversas" juegan un papel determinante en los resultados en salud. Sin embargo, a diferencia de los factores socioestructurales, en los factores psicosociales de la salud cobra especial importancia la subjetividad colectiva. Numerosas investigaciones han mostrado que las personas expuestas a eventos estresantes tienen un mayor riesgo de sufrir trastornos psicológicos y psiquiátricos, empobrecimiento de la salud física y/o abuso de sustancias ${ }^{(77)}$. Mientras gran parte de la investigación ha puesto el foco de atención en la exposición a determinados eventos, otros estudios han subrayado el papel de los "estresores crónicos"(78).

Desde una perspectiva crítica, el debate sobre el impacto potencial de los factores psicosociales es uno de los más controvertidos en la investigación sobre las desigualdades en salud. En el caso de las masculinidades, las formas en que dichos factores traspasan el tamiz del género han sido escasamente tenidas en cuenta. Desde este planteamiento, 
el efecto en los hombres -de considerarse económica y socialmente inferiores (con relación a otros hombres pero, especialmente, en el caso de parejas heterosexuales, con relación a sus parejas) - suele asociarse a una disminución en los niveles de autoestima; algo que, directa o indirectamente, afecta a la percepción de bienestar, además de contribuir a determinadas prácticas de riesgo, tales como el consumo abusivo de sustancias psicoactivas ${ }^{(79)}$. La investigación científica también apunta a otra área de estudio de gran interés, como la interacción del género con salud mental y desempleo. Algunos estudios han descrito cómo la asunción del rol de sustentador principal a nivel familiar podría ejercer en los hombres un efecto negativo superior sobre su salud en caso de tener que enfrentar una situación de pérdida de empleo. En el caso del estudio realizado por Artazcoz et al. ${ }^{(66)}$ las responsabilidades familiares asumidas por las mujeres, especialmente en lo concerniente a la crianza de hijos e hijas, ejercían un efecto amortiguador en el impacto negativo en la salud mental tras la pérdida del empleo, efecto que no se presente en la misma medida en los hombres.

Uno de los valores añadidos de la incorporación de las explicaciones psicosociales a los estudios sobre la salud de los hombres tiene que ver con observar de forma simultánea los efectos psicosociales atendiendo a distintas "jerarquías". Esto implica que el impacto de las masculinidades puede ser visto, por ejemplo, con relación a la clase social o el grupo étnico de pertenencia (lo que supone un guiño conceptual a la perspectiva interseccional). Esto también permite describir ciertos comportamientos negativos para la salud como una forma de agencia desde la que superar otras formas de desigualdad presentes entre distintos grupos de hombres. En cualquier caso, no hay que olvidar que, desde un enfoque relacional, el género se construye fundamentalmente a través de la interacción personal ${ }^{(80)}$, algo que ha llevado también a subrayar su sentido performativo. Es decir, el género entendido como una entidad que implica una repetición de actuaciones que definen la experiencia compartida; estamos ante un componente característico en el análisis de los hombres, que permite describir los "actos de masculinidad" como una forma de subjetividad que, no solo genera un sentido de pertenencia grupal, sino que también contribuye a la explicación respecto de adoptar/ evitar determinados riesgos ${ }^{(81)}$.

\section{Explicaciones desde el enfoque del ciclo vital}

El enfoque del ciclo vital incorpora elementos tanto del materialismo como de las explicaciones culturales y psicosociales, pero extiende la cadena causal de dichas explicaciones. De forma general, sugiere que el estado de salud, en una determinada edad y para una cohorte concreta, no solo refleja las condiciones actuales, sino también las circunstancias vitales anteriores ${ }^{(82)}$. Sin embargo, el enfoque del ciclo vital no supone simplemente la recolección de datos a lo largo del curso de toda una vida, sino que implica la comprensión del ordenamiento temporal de las variables de exposición y sus interrelaciones ${ }^{(83)}$. Dentro de la salud pública, uno de los modelos más recurrentes ha sido el de "efectos acumulativos", según el cual la intensidad o la duración de la exposición a ambientes desfavorables en diferentes etapas de la vida tienen un efecto adverso acumulativo en la salud. En torno a esta línea, han sido especialmente prolíficos los estudios que han mostrado cómo el efecto acumulativo de las condiciones de vida con bajos ingresos, en combinación con otros indicadores de pobreza, como el bajo nivel educativo o las malas condiciones laborales, da lugar a importantes desigualdades en salud ${ }^{(84)}$.

Una constante en el estudio de los hombres y las masculinidades ha sido el énfasis en explorar las dinámicas sociales en torno a las cuales las identidades son "(re)negociadas $^{\prime \prime(85)}$. Es por ello que, recientemente, algunos de los enfoques teóricos más influyentes a la hora de abordar la salud de los hombres hayan subrayado la necesidad de incorporar una perspectiva centrada en el ciclo vital. Esto está permitiendo establecer sinergias positivas entre la investigación orientada por 
este enfoque y los estudios de la salud de los hombres desde una perspectiva crítica. En el desarrollo de este enfoque son de gran relevancia los estudios longitudinales, basados en el seguimiento de los procesos de saludenfermedad en diferentes etapas del ciclo vital. Existe un importante corpus de conocimiento sobre diferencias en los resultados en salud de mujeres bajo determinadas circunstancias sociales, como, por ejemplo, en relación con su estado civil, su participación en el ámbito laboral o la edad de sus hijos e hijas $^{(86)}$. Sin embargo, en el caso de los hombres, son escasos los estudios centrados en transiciones como la paternidad o la jubilación, en los que el análisis centrado estrictamente en el criterio edad puede no captar en toda su extensión las dinámicas que se generan en torno a dichos procesos ${ }^{(87)}$. De esta manera, la incorporación del enfoque del ciclo vital debería facilitar una visión más profunda del efecto acumulativo de las experiencias dentro de un "entorno relacional". Esto fortalecería el entendimiento de cómo las concepciones hegemónicas de la masculinidad resisten/cambian a lo largo del tiempo.

\section{REFLEXIONES FINALES: A MODO DE AGENDA PARA SEGUIR AVANZANDO}

Poner el foco de atención en la relación entre género y equidad ha ido progresivamente introduciendo la cuestión de las masculinidades en el debate sobre los determinantes sociales de la salud, aspecto íntimamente conectado al desarrollo de la nueva agenda en salud pública. Esto viene conformando un campo de estudio que se ha visto favorecido por el desarrollo de los distintos enfoques explicativos de las desigualdades de género en salud, que no deberían concebirse como interpretaciones contrapuestas a la hora de integrarlos en el estudio de los hombres y las masculinidades. Esto nos lleva a formular una serie de recomendaciones orientadas a las políticas y la investigación en salud pública, que consideramos pueden contribuir a seguir avanzando en el estudio y el desarrollo de programas desde una perspectiva de género en salud.

\section{Recomendaciones orientadas a las políticas}

Aunque la mayor brecha en morbilidad entre hombres y mujeres se debe a diferencias comportamentales, enfocar el diseño de programas y políticas exclusivamente sobre la base de los comportamientos de riesgo de los hombres, contribuye a fortalecer un abordaje de género conceptualmente restrictivo. De ahí, por ejemplo, la relevancia de poner en evidencia las relaciones que se establecen entre las distintas concepciones de la masculinidad y la exposición a factores socioeconómicos y psicosociales. Del mismo modo, se hace necesario hacer un reconocimiento de las desigualdades en salud existentes entre los propios hombres. Abordar la salud de los hombres como si se tratara de un grupo homogéneo implica no tener en cuenta la heterogeneidad de sus experiencias vitales, lo que contribuye a no promocionar otras formas más positivas de masculinidad. Impulsar, desde una perspectiva de género, un movimiento en favor de la salud de los hombres, integrado con el de las mujeres, debe comenzar por un reconocimiento de las complejidades en la conformación de las identidades masculinas.

Debemos desarrollar y evaluar políticas y ejemplos de buenas prácticas dirigidas a promover la salud de los hombres de una manera que también contribuya a mejorar la de las mujeres. Esto es particularmente necesario en relación con las responsabilidades dentro de la esfera doméstica, promoviendo que los hombres asuman la responsabilidad del cuidado informal de familiares dependientes o una mayor implicación en la crianza de sus hijos e hijas.

Consideramos que también es necesario desarrollar y difundir más ejemplos de cómo la interseccionalidad y la incorporación de la perspectiva de género se pueden aplicar de una manera pragmática, con especial interés en los hombres que muestran una mayor 
conformidad con los ideales tradicionales de masculinidad o con identidades no normativas. En cualquier caso, los programas de promoción de la salud y las intervenciones específicas no deben limitarse exclusivamente a abordar factores de riesgo, sino a construir salud desde una perspectiva de género, partiendo del reconocimiento de formas positivas de desarrollar la identidad masculina. Reconocer las consecuencias de las formas hegemónicas de masculinidad para la salud de hombres (y mujeres) no debería convertirse, simplemente, en una forma de culpabilización, sino un aspecto que debe guiar el desarrollo de políticas de salud más integrales y sensibles respecto a la incorporación de la perspectiva de género.

\section{Recomendaciones orientadas a la investigación}

Se hace necesario potenciar una visión comparativa y colaborativa a la hora de abordar la salud de mujeres y hombres. Esto requiere no analizar los procesos de salud/enfermedad como meros procesos antagónicos. Es necesario el desarrollo de líneas de investigación centradas en proporcionar evidencia, cuando exista, sobre sobre las interacciones sexo-género y sus efectos en la salud de las personas. Además, un enfoque relacional facilita el estudio de los estereotipos, valores y prácticas cotidianas relacionadas con las diferencias/semejanzas en la exposición, no solo a factores de riesgo, sino también a condiciones o factores generadores de salud. Esto contribuiría a mejorar el diseño de las actuaciones preventivas y de promoción de la salud.

Del mismo modo, los estudios que han analizado la salud de los hombres dentro de diferentes grupos socioeconómicos son aún muy limitados. Sería de gran interés potenciar el desarrollo de estudios centrados en la intersección entre las prácticas sociales hegemónicas y las prácticas institucionalizadas como, por ejemplo, tener que realizar largas jornadas de trabajo o realizar horas extraordinarias; situaciones con consecuencias para la salud, que contribuyen a reforzar ciertos roles sociales entre hombres y mujeres.

Se requiere un análisis más detallado de las circunstancias y las condiciones de vida de aquellos hombres que mantienen y ensalzan actitudes y prácticas que ponen en riesgo su salud. El desarrollo de metodologías de mediación e integración sociocomunitaria de investigación-acción-participativa podría ser de gran interés para evidenciar otras formas de entender la masculinidad, incluyendo las relacionadas con la diversidad afectivosexual e identidades de género, y contribuir a visibilizar aquellas manifestaciones pertenecientes a un ideario de masculinidad más positivo. En este sentido, resulta de especial interés estudiar cómo las concepciones no hegemónicas benefician la salud de los propios hombres, y cómo esto repercute también sobre las de las mujeres.

\section{REFERENCIAS BIBLIOGRÁFICAS}

1. Risman RJ, Davis G. From sex roles to gender structure. Current Sociology Review. 2013;61(56):733-755.

2. Connell RW. Gender and Power: Society, the Person and Sexual Politics. Standford CA: Standford University Press; 1987.

3. Carabí A. Construyendo nuevas masculinidades: una introducción. En: Segarra M, Carabí A. Nuevas masculinidades. Barcelona: Icaria Editorial; 2000. p. 15-27.
4. Rubin G. El tráfico de mujeres: notas sobre la "economía política" del sexo. Nueva Antropología. 1986;8(30):95-145.

5. Butler J. El género en disputa: el feminismo y la subversión de la identidad. Barcelona: Paidós; 2007.

6. Del Valle T. Perspectivas feministas desde la antropología social. Barcelona: Editorial Ariel; 2000.

7. World Health Organization. The health and well-being of men in the WHO European Region: better health through a gender approach [Internet]. 
2018 [citado 28 mar 2019]. Disponible en: https:// tinyurl.com/wvowupg.

8. Esteban ML. El estudio de la salud y el género: las ventajas de un enfoque antropológico y feminista. Salud Colectiva. 2006;2(1):9-20.

9. Sabo D. The study of masculinities and men's health: An overview. En: Kimmel EM, Hearn J, Connell RW, (ed.). Handbook of studies on men and masculinities. Thousand Oaks, CA: Sage; 2005. p. 326-353.

10. Emslie C, Hunt K. Live to work or work to live? A qualitative study of gender and work-life balance among men and women in mid-life. Gender, Work and Organization. 2009;16(1):151-72.

11. Annandale E, Hunt K. Gender inequalities in health. Buckingham, Philadelphia: Open University Press; 2000.

12. Connell RW. Gender: In world perspective. Cambridge: Polity; 2009.

13. Iyer A, Sen G, Östlin P. Inequalities and intersections in health: a review of the evidence. En: Sen EG, Östlin P, (ed.). Gender equity in health: The shifting frontiers of evidence and action. New York: Routledge; 2010.

14. Annandale E, Riska E. New connections: Towards a gender-inclusive approach to women's and men's health. Current Sociology. 2009;57(2):123-133.

15. World Health Organization (WHO). What about boys? A literature Review on the Health and Development of Adolescent Boys [Internet]. 2000 [citado 28 mar 2019]. Disponible en: https:// tinyurl.com/t42pr7w.

16. Meryn S, Jadad AR. The future of men and their health. Are men in danger of extinction?. British Medical Journal. 2001;323(7320):1013-1014.

17. Commission on Social Determinants of Health $(\mathrm{CSDH})$. Achieving Health Equity: from root causes to fair outcomes. [Internet]. 2007 [citado $28 \mathrm{mar}$ 2019]. Disponible en: https://tinyurl.com/v6rkn4f.

18. Naciones Unidas. Comisión de la Condición Jurídica y Social de la Mujer Informe sobre el 54o período de sesiones [Internet]. 2010 [citado 28 mar 2019]. Disponible en: https://tinyurl.com/ugulbwz.

19. Kleinman A. Four social theories for global health. The Lancet. 2010;375(9725):1518-1519.

20. Blaxter M. Who fault is it? People's own conceptions of the reason for health inequalities. Social Science \& Medicine. 1997;44(6):747-756.
21. Krieger N. Genders, sexes, and health: What are the connections-and why does it matter? International Journal of Epidemiology. 2003;32(4):652657.

22. Popay J, Williams G. Equalizing the people's health: a sociological perspective. En: Gabe EJ, Calnan M, (ed.). The new sociology of the health service. London: Routledge; 2009. p. 222-244.

23. Lock M, Freeman J, Chilibeck G, Beveridge B, Padolsky M. Susceptibility genes and the question of embodied identity. Medical Anthropology Quarterly. 2007;21(3):256-276.

24. Backhans M, Burström B (dir). Gender policy and gender equality in a public health perspective: Investigating morbidity and mortality in Sweden and 22 OECD countries [Tesis de Doctorado]. Estocolmo: Karolinska Institute; 2011[citado 28 mar 2019]. Disponible en: http://hdl.handle.net/10616/40644.

25. Riska E. Women's health: issues and prospects. Scandinavian Journal of Public Health. 2000;28:8487.

26. Farrell W. The liberated man: Beyond masculinity. New York: Random House; 1975.

27. Feigen Fasteau M. The male machine. New York: McGraw-Hill; 1974.

28. Ruiz-Cantero MT, Vives-Cases C, Artazcoz L, Delgado A, García-Calvete MM, Miqueo C, et al. A framework to analyse gender bias in epidemiological research. Journal of Epidemiology \& Community Health. 2007;61(2):46-53.

29. Read JG, Gorman BK. Gender and health inequality. Annual Review of Sociology. 2010;36:371-386.

30. Verbrugge LM. Gender and health: an update on hypotheses and evidence. Journal of Health \& Social Behavior. 1985;26(3):156-182.

31. Centers for Disease Control and Prevention (CDC). Morbidity and Mortality Weekly Reports from 1994 [Internet]. 1994 [citado 28 mar 2019]. Disponible en: https://tinyurl.com/uewalg3.

32. Connell RW. Gender, health and theory: conceptualizing the issue, in local and world perspective. Social Science \& Medicine. 2012;74:1675-1683.

33. Braveman P. Health Disparities and Health Equity: Concepts and Measurement. Annual Review of Public Health. 2006;27:167-194.

34. Hammarström A, Härenstam A, Östlin P. Gender and health: concepts and explanatory models. En: Piroska Ö, Danielson M, Finn D, Härens- 
tem A, Lindberg G, (ed.). Gender inequalities in health: A Swedish perspective. Cambridge, MA: Harvard Centre for Population and Development Studies; 2001.

35. Ruiz-Cantero MT, Verbrugge LM. A two way view of gender bias in medicine. Journal of Epidemiology and Community Health. 1997;51(2):106-9.

36. Owens IPF. Sex differences in mortality rate. Science. 2002;297:2008-09.

37. GBD 2017 Mortality Collaborators. Global, regional, and national age-sex-specific mortality and life expectancy, 1950-2017: a systematic analysis for the Global Burden of Disease Study 2017. The Lancet. 2018;392:1684-1735.

38. Bird CE, Rieker PP. Gender and health: The effects of constrained choices and social policies. New York: Cambridge University Press; 2008.

39. Phillips SP. Including gender in public health research. Public Health Reports. 2011;126(3):16-21.

40. European Commission. The State of Men's Health in Europe [Internet]. 2011 [citado 28 mar 2019]. Disponible en: https://tinyurl.com/qooz5tw.

41. World Health Organization. Women's health and well-being in Europe: beyond the mortality advantage [Internet]. 2016 [citado 28 mar 2019]. Disponible en: https://tinyurl.com/w4meey7.

42. Verbrugge LM. The twain meet: Empirical explanations of sex differences in health and mortality. Journal of Health \& Social Behavior. 1989;30:282-304.

43. Sen G, Östlin P. Unequal, unfair, ineffective and inefficient. Gender inequity in health: why it exists and how can change it. [Internet]. 2007 [citado 28 mar 2019]. Disponible en: https://tinyurl. com/yx8kzzn2.

44. Doyal L. Gender equity in health: debates and dilemmas. Social Science \& Medicine. 2000;51:931939.

45. Lupton D. Risk as moral danger: The social and political functions of risk discourse in public health. International Journal of Health Services. 1993;23(3):425-435.

46. Solar O, Irwin A, Vega J. Determinants of health disease: overview and framework. En: Detels R, Beaglehole R, Lansang MA, Gulliford M, (ed.). Textbook of Public Health. Oxford: Oxford University Press; 2009. p. 101-119.
47. Perry DG, Pauletti RE. Gender and Adolescent Development. Journal of Research on Adolescence. 2011;21(1):61-74.

48. Robertson S. Understanding men and health: Masculinities, identity and well-being. London: Open University Press; 2007.

49. de Visser RO. I'm not a very manly man. Qualitative insights into young men's masculine subjectivity. Men and Masculinities. 2009;11(3):367-371.

50. Measham F. "Doing gender"-"doing drugs": Conceptualising the gendering of drug cultures. Contemporary Drug Problems. 2002;29(2):335-373.

51. Kimmel MS. Homofobia, temor, vergüenza y silencio en la identidad masculina. En: Valdés ET, Olavarría J, (ed.). Masculinidad/es: poder y crisis. Santiago: ISIS-FLACSO/Ediciones de las Mujeres No24; 1997. p. 49-62.

52. Courtenay WH. Constructions of masculinity and their influence on men's well-being: a theory of gender and health. Social Science \& Medicine. 2000;50(10):1385-1401.

53. Evans J, Frank B, Oliffe JL, Gregory D. Health, Illness, Men and Masculinities (HIMM): a theoretical framework for understanding men and their health. Journal of Men's Health \& Gender. 2011;8(1):7-15.

54. Vandello JA, Bosson JK. Hard won and easily lost: a review and synthesis of theory and research on precarious manhood. Psychology of Men \& Masculinity. 2013;14(2):101-113.

55. Martín-Criado E. Producir la juventud. Crítica de la sociología de la juventud. Madrid: Istmo; 1998.

56. Lupton D, Tulloch J. "Risk is part of your life": Risk Epistemologies among a Group of Australians. Sociology. 2002;36(2):317-334.

57. France A. Towards a Sociological Understanding of youth and their risk-taking. Journal of Youth Studies. 2000;3(3):317-331.

58. Holmes D, Gastaldo D, O'Byrne P, Lombardo A. Bareback Sex: A Conflation of Risk and Masculinity. International Journal of Men's Health. 2008;7(2):171-191.

59. Marston C, King E. Factors that shape young people's sexual behaviour: a systematic review. The Lancet. 2006;368:1581-1586. 
60. Sen A. Inequality reexamined. Cambridge, MA: Harvard University Press; 1992.

61. Kimmel MS, Hearn J, Connell RW. Handbook of studies on men and masculinities. London: Sage; 2005.

62. Kawachi I, Subramanian SV, Filho A, N. A glossary for health inequalities. Journal of Epidemiology \& Community Health. 2002;56(9):647-652.

63. Navarro V. The political and social context of health. New York: Baywood Publishing Company; 2004.

64. Benach J, Muntaner C, Solar O, Santana V, Quinlan M. Empleo, trabajo y desigualdades en salud: una visión global. Barcelona: Icaria Editorial; 2010.

65. Backhans MC, Lundberg $M$, Mänsdotter A. Does increased gender equality lead to a convergence of health outcomes for men and women?: A study of Swedish municipalities. Social Science \& Medicine. 2007;64(9):1892-1903.

66. Artazcoz L, Benach J, Borrell C, Cortés I. Unemployment and mental health: Understanding the interactions among gender, family roles, and Social Class. American Journal of Public Health. 2004;94(1):82-88.

67. Organización Internacional del Trabajo (OIT). World employment social outlook: trends for women 2018, global snapshot. [Internet]. 2018 [citado 28 mar 2019]. Disponible en: https:// tinyurl.com/ycw42qay.

68. Craig L, Sawrikar P. Work and family: How does the (gender) balance change as children grow? Gender, Work and Organization. 2009;16(6):684709.

69. Larrañaga I, Martín U, Bacigalupe A, Begiristáin JM, Valderrama MJ. Impacto del cuidado informal en la salud y la calidad de vida de las personas cuidadoras: análisis de las desigualdades de género. Gaceta Sanitaria. 2008;22(5):443-450.

70. Fuhrer R, Stansfeld SA. How gender affects patterns of social relations and their impact on health: a comparison of one or multiple source of support from. Social Science \& Medicine. 2002;54:811-825.

71. Chen YY, Subramanian SV, Acevedo-García D, Kawachi I. Women's status and depressive symptoms: A multilevel analysis. Social Science \& Medicine. 2005;60:49-60.

72. Masanet E, La Parra D. Relación entre el número de horas de cuidado informal y el estado de salud mental de las personas cuidadoras. Revista Española de Salud Pública. 2011;85:257-266.

73. Van de Velde $S$, Bracke $P$, Levecque $K$. Gender differences in depression in 23 European countries: Cross-national variation in the gender gap in depression. Social Science \& Medicine. 2010;71(2):305-313.

74. García-Calvente MM. del Rio-Lozano M, Marcos-Marcos J. Desigualdades de género en el deterioro de la salud como consecuencia del cuidado informal en España. Gaceta Sanitaria. 2011;25(S2):100-107.

75. Neff LA, Karney BR. To know you is to love you: The implications of global adoration and specific accuracy for marital relationships. Journal of Personality and Social Psychology. 2005;88(3):480-497.

76. Adamson JA, Ebrahim S, Hunt K. The psychosocial versus material hypothesis to explain observed inequality in disability among older adults: Data from the West of Scotland Twenty-07 Study. Journal of Epidemiology and Community Health. 2006;60(11):974-980.

77. Schneiderman N, Ironson G, Siegel SD. Stress and health: Psychological, behavioral, and biological determinants. Annual Review of Clinical Psychology. 2005;1:607-628.

78. McDonough P, Walters V. Gender and health: reassessing patterns and explanations. Social Science \& Medicine. 2001;52:547-559.

79. Robertson S. Men managing health. Men's Health Journal. 2003;2(4):111-113.

80. West C, Zimmerman DH. Accounting for doing gender. Gender \& Society. 2009;23(1):112-122.

81. Marcos-Marcos J, Romo-Avilés N, del RíoLozano M, Palomares-Cuadros J, García-Calvete MM. Performing masculinity, influencing health: a qualitative mixed-methods study of Spanish young men. Global Health Action. 2013;6:1.

82. Graham H. Building an Inter-disciplinary science of health inequalities: The example of life-course research. Social Science \& Medicine. 2002;55(11):2006-2016.

83. Ben-Shlomo Y, Kuh D. A lifecourse approach to chronic disease epidemiology: Conceptual models, empirical challenges and interdisciplinary perspectives. International Journal of Epidemiology. 2002;31(2):285-293.

84. Arber S, Thomas H. From women's health to a gender analysis of health. En: Cockerham WC, (ed.). 
The Blackwell companion to medical sociology. Malden, MA: Blackwell Publishing; 2001. p. 94-113.

85. Connell RW. Masculinities. Cambridge: Polity Press in association with Blackwell; 1995.

86. Artazcoz L, Cortés I, Borrell C, Escribá-Agüir $\mathrm{V}$, Cascant L. Social inequalities in the association between partner/marital status and health among workers in Spain. Social Science \& Medicine. 2011;72(4):600-607.

87. Lohan M. How might we understand men's health better? Integrating explanations from critical studies on men and inequalities in health. Social Science \& Medicine. 2007;65:493-504.

\section{FORMA DE CITAR}

Marcos-Marcos J, Mateos JT, Gasch-Gallén A, Álvarez-Dardet C. El estudio de la salud de los hombres desde una perspectiva de género: de dónde venimos, hacia dónde vamos. Salud Colectiva. 2020;16:e2246. doi: 10.18294/ sc. 2020.2246 .

Recibido: 29 mar 2019 | Aprobado: 6 mar 2020 | Publicado en línea: 24 abr 2020

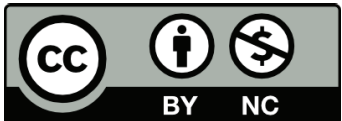

Esta obra está bajo una licencia de Creative Commons Reconocimiento-NoComercial 4.0 Internacional. Reconocimiento - Permite copiar, distribuir y comunicar públicamente la obra. A cambio, se debe reconocer y citar al autor original. No Comercial - Esta obra no puede ser utilizada con finalidades comerciales, a menos que se obtenga el permiso. 